ARTICLE

Received 9 Jan 2015 | Accepted 20 Oct 2015 | Published 30 Nov 2015

DOl: $10.1038 /$ ncomms 9945

OPEN

\title{
Adaptive immunity increases the pace and predictability of evolutionary change in commensal gut bacteria
}

João Barroso-Batista', Jocelyne Demengeot ${ }^{1} \&$ Isabel Gordo ${ }^{1}$

Co-evolution between the mammalian immune system and the gut microbiota is believed to have shaped the microbiota's astonishing diversity. Here we test the corollary hypothesis that the adaptive immune system, directly or indirectly, influences the evolution of commensal species. We compare the evolution of Escherichia coli upon colonization of the gut of wild-type and Rag $2^{-/-}$mice, which lack lymphocytes. We show that bacterial adaptation is slower in immune-compromised animals, a phenomenon explained by differences in the action of natural selection within each host. Emerging mutations exhibit strong beneficial effects in healthy hosts but substantial antagonistic pleiotropy in immune-deficient mice. This feature is due to changes in the composition of the gut microbiota, which differs according to the immune status of the host. Our results indicate that the adaptive immune system influences the tempo and predictability of $E$. coli adaptation to the mouse gut.

\footnotetext{
${ }^{1}$ Instituto Gulbenkian de Ciência, Rua da Quinta Grande 6, 2780-156 Oeiras, Portugal. Correspondence and requests for materials should be addressed to I.G. (email: igordo@igc.gulbenkian.pt).
} 
T he maintenance of a healthy status in mammals depends on the interaction between their microbiota and the immune system. The partnership between gut commensals and the host is hypothesized to result from millions of years of co-evolution, where the host immune response is tightly regulated to tolerate commensals, which in turn shape its development. Indeed, it recently became clear that specific bacterial species, such as segmented filamentous bacteria ${ }^{1}$, Clostridia spp. ${ }^{2}$ and Bacteroides fragilis ${ }^{3}$, can promote the expansion of regulatory and pro-inflammatory $\mathrm{T}$ cells, promoting immune homeostasis and contributing to restoration of health. Further support for a role of microbes in controlling host physiology comes from faecal transplants, which can affect their weight ${ }^{4}$ and even protect hosts from invasion of pathogens ${ }^{5}$. The patterns of microbiota species composition also differ between healthy and immunecompromised mice lacking an adaptive immune system ${ }^{6,7}$, suggestive of the hypothesis of co-evolution between host and gut commensals 8 . However, a direct support for co-evolution requires the study of how host genetics affects evolutionary change within the gut microbial species it carries and vice versa. These issues have been poorly studied, probably partly due to the difficulty in conducting studies that demonstrate the direct influence of microbial evolution on mammalian host evolution. It is, however, easier to address the influence of host genetics on microbial ecology and evolution.

Escherichia coli, the most abundant aerobe and one of the first species to colonize the human gut, offers a powerful system to investigate how and to what extent the genetic composition of a commensal changes with time when facing a healthy or a compromised host immune system. For example, in patients with inflammatory bowel diseases, such as Crohn's disease and ulcerative colitis, which are increasing in incidence in the human population and are associated with different mutations in genes with important immune functions, often show an increased abundance of Proteobacteria, namely E. coli, which tends to be sampled, in comparison with healthy hosts ${ }^{9,10}$.

Using a classical E. coli colonization system-the streptomycintreated mouse-we have recently shown that $E$. coli undergoes rapid adaptation to the intestinal tract ${ }^{11}$. This adaptation was detected through observation of phenotypic sweeps, which occurred across populations of $E$. coli evolving independently in genetically identical immune-competent hosts. Here, we have tested the hypothesis that the process of adaptation of $E$. coli can be altered in immune-compromised mice lacking an adaptive immune system $\left(\right.$ Rag $\left.2^{-1-}\right)$. We show that evolution of $E$. coli is slower in these hosts than in immune-competent animals, due to smaller selective effects of the first beneficial mutations that emerge in these bacteria. We also demonstrate that the strength of natural selection is dependent on the composition of the microbiota, which differs between immune-competent and immune-compromised animals. Furthermore, we describe the genetic targets of E. coli adaptation and find adaptive mutations specific to the host genetic background. Finally, the findings that emerging mutations exhibit strong beneficial effects in healthy hosts but substantial antagonistic pleiotropy in immune-deficient mice, support the notion that the adaptive immune system enhances the predictability of adaptive evolution of bacteria comprising the microbiota. Together, these results indicate that not only the microbiotic environment but also the pace and the path of adaptation of a commensal species can be altered by the host immune system.

\section{Results}

E. coli adaptation is slower in immune-compromised mice. To study E. coli adaptation in the gut of Rag2 ${ }^{-1-}$ mice, we colonized 15 animals (see Methods) with two E. coli strains, isogenic except for the presence of a neutral fluorescent marker (cyan fluorescent protein (CFP) and yellow fluorescent protein (YFP)). We first measured the frequency of the neutral marker and its dynamics by daily monitoring of $E$. coli numbers and fluorescence in the faecal content. Much smaller changes in marker frequency were detected in Rag2 $2^{-1-}$ compared with wild-type (WT) mice ${ }^{11}$ during the first 6 days of colonization (Fig. 1a, binomial test $P=0.03$ ), suggesting a slower rate of adaptation in immune-compromised hosts. This delayed evolutionary change was not due to smaller population size, as similar loads of E. coli were recovered from both animals (Supplementary Fig. 1). After this initial period, the marker frequency started to diverge (Fig. 1b) in some Rag2 $2^{-1-}$ animals, as had been observed in WT mice ${ }^{11}$. Some populations showed a signature of a selective sweep, where a single marker steadily increased towards fixation (lines R1.1 and R1.12). Other populations showed the classical signature of clonal interference (R1.11 and R1.15), where clones carrying a given fluorescence first increased in frequency and then were replaced by clones with a different fluorescence, likely due to beneficial mutations with stronger effect arising in the latter. We next asked whether the slower pace of adaptation in Rag2 ${ }^{-1-}$ mice could be due to a longer time for sweeps to occur. In WT mice, the first adaptive mutations to appear involve changes in genes of the gat operon, conferring $E$. coli with a gat-negative phenotype ${ }^{11}$. In Rag2 ${ }^{-1-}$ mice the same phenotype emerged and swept (Supplementary Fig. 2; Fig. 1c), but at a slower pace $\left(\mathrm{z}=-25.5, P<10^{-3}\right)$. Though mutations in this operon ultimately reached high frequency in the majority of lineages evolving in Rag2 ${ }^{-}$ mice, they could only be detected after 3 days of adaptation. This contrasts with what was seen in WT mice. This occurred within the same experimental groups (Supplementary Fig. 2, upper panels versus lower panels). Although we detected populations where the increase in frequency of the phenotype was as fast as in WT (Supplementary Fig. 2, lines R1.3 and R1.10), this increase was slower in the majority of the populations. Thus, the gatnegative phenotype arose faster (Fig. 1c), and variance in this phenotype was eliminated quicker (Fig. 1d), in WT compared with $\mathrm{Rag}^{-1-}$ animals. Both patterns are consistent with faster adaptation under the pressures of an adaptive immune system.

Duplication time of $\boldsymbol{E}$. coli in the mouse gut. We then sought to identify the mechanism responsible for the observed slower adaptive pace in Rag2 ${ }^{-1-}$ mice. Adaptation rate depends on generation time, population size, mutation rate and the strength of selection on newly adaptive alleles ${ }^{12}$. Population size estimates based on bacterial loads recovered from both groups of animals (Supplementary Fig. 1) suggested that this factor would not play a leading role in the observed differences. To determine the division rate of $E$. coli in the mouse gut, we used in situ hybridization with a probe specific for E. coli $23 S$ ribosomal RNA (rRNA) to estimate cellular rRNA content, which strongly correlates with bacterial division rate (Supplementary Fig. 3), using an adapted version of a previously described method ${ }^{13}$. We colonized WT and Rag2 ${ }^{-1-}$ mice with E. coli and collected faecal samples at days 1 and 3 after inoculation, when E. coli had already reached the same load as observed during the course of the evolution experiment (Supplementary Fig. 1). On the basis of the fluorescence intensity of hybridized $E$. coli cells, we inferred an average duplication time of $66( \pm 3,2$ s.e.m.) and $76( \pm 3,2$ s.e.m.) min in Rag2 ${ }^{-1-}$ and WT mice, respectively (Fig. 2a). As the generation time for E. coli was significantly smaller in Rag2 ${ }^{-1}$ - than in WT mice (Mann-Whitney $U$-test, $W=111.5$, $\left.P<10^{-3}\right)$, this parameter also could not explain the different rates of adaptation observed between hosts. 
a

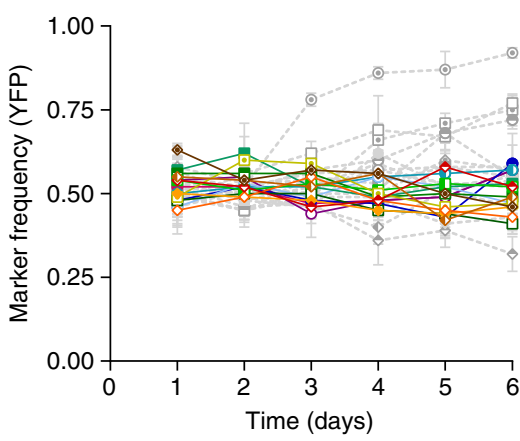

C

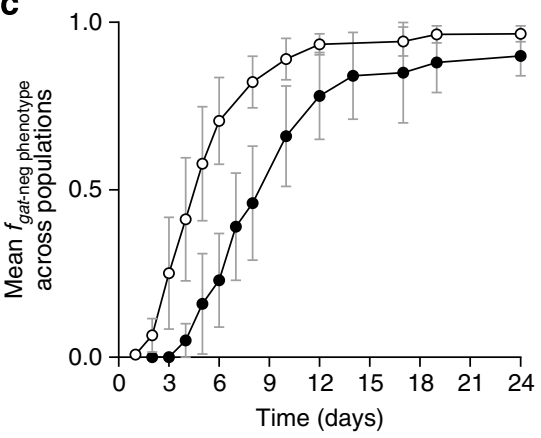

b

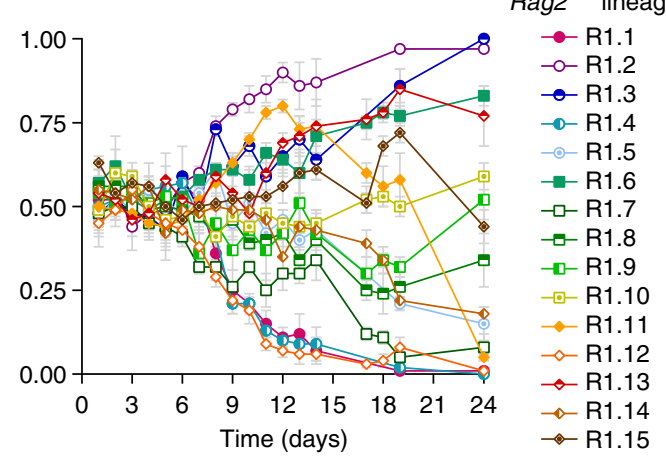

d

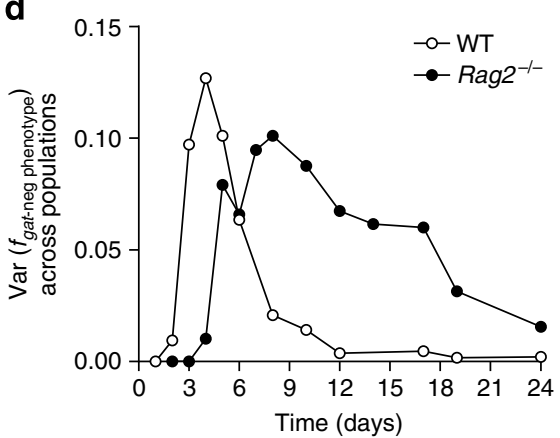

Figure 1 | E. coli adapts at a slower pace in immune-compromised mice. Dynamics of marker frequency $( \pm 2$ s.e.m., representing the error associated with the sampling when calculating the frequency of the marker) during the first 6 (a) or 24 (b) days of adaptation of E. coli upon colonization of Rag $2^{-/-}$ (coloured) or WT (grey) mice (reproduced from ref. ${ }^{11}$ for clarity). (c) Average frequency of the gat-negative phenotype ( \pm 2 s.e.m.) over time across WT (white) and Rag2 ${ }^{-/-}$(black) animals ( $n=15$, for each host genotype) and the variance for the frequency (d).

Estimation of the $E$. coli mutation rate in the mouse gut. Having observed a shorter E. coli duplication time in Rag2 - Icompared with WT animals, we tested the hypothesis that the observed delay in the emergence of the adaptive phenotype in Rag2 $2^{-1-}$ mice was due to a lower spontaneous mutation rate in these hosts. We determined the spontaneous mutation frequency towards resistance to different antibiotics, by measuring the number of clones resistant to rifampicin, nalidixic acid or furazolidone in E. coli populations colonizing Rag $2^{-I-}$ and WT mice over a period of 15 days (Fig. 2b). Resistance against these antibiotics can be conferred through mutations in specific $E$. coli genes ( $r p o B, g y r A$ and $n f s A$, respectively). Resistance to rifampicin $\left(\mathrm{Rif}^{\mathrm{R}}\right)$ and nalidixic acid $\left(\mathrm{Nal}^{\mathrm{R}}\right)$ involves mainly point mutations, while resistance to furazolidone $\left(\mathrm{Fzd}^{\mathrm{R}}\right)$ can also be acquired by transposition of insertion sequence (IS) elements ${ }^{14}$. Assuming that such resistant alleles are slightly deleterious, and thus kept at mutation-selection balance in a large population, the fraction of resistant mutants is proportional to the mutation rate $^{12}$. We estimated an average $\log _{10}$ mutation frequency for $\mathrm{Rif}^{\mathrm{R}}$ of -7.53 in $R a g 2^{-1-}$ animals, not significantly different from that in WT mice (Mann-Whitney $U$-test, $W=284.5, P=0.95)$. A similar result was found for $\mathrm{Nal}^{\mathrm{R}}$ mutants $(-7.76$ in WT and -7.76 in Rag2 $2^{-1-}$ mice, Mann-Whitney $U$-test, $\left.W=202, P=0.65\right)$ and $\mathrm{Fzd}^{\mathrm{R}}\left(-5.65\right.$ in $\mathrm{WT}$ and -5.49 in Rag2 ${ }^{-1-}$ mice, Mann-Whitney $U$-test, $W=1613, \quad P=0.10)$. We also measured the in vivo frequency of spontaneous resistant mutants to furazolidone, where resistance was achieved through transpositions, therefore providing the first estimate of the in vivo spontaneous transposition frequency. This is an important parameter in E. coli adaptation to the gut, given that approximately half of the adaptive mutations identified in WT mice were caused by insertion of transposable elements ${ }^{11}$. We estimated an average $\log _{10}$ transposition frequency of -5.99 in WT and a similar frequency of -5.75 in $R a g 2^{-1-}$ mice (Mann-Whitney $U$-test, $W=83, P=0.09$ ). Taken together, these results indicate that both the point-mutation frequency and transposition frequency are similar in immune-compromised and immune-competent animals, suggesting that the slower rate of adaptation observed in the former was not due to an overall decreased mutation rate of $E$. coli.

Altered selective pressures in immune-compromised mice. We next asked whether the fitness effects of adaptive mutations were different in the gut of $R a g 2^{-1-}$ and WT mice. We determined the fitness effect $(s)$ of a mutant (gat $Z$ ) harbouring a single beneficial mutation through in vivo competition assays against the ancestral E. coli (see Methods). In WT mice (Fig. 2c, left panel; Supplementary Table 1), we estimated a mean advantage, per hour $\left(s_{\text {gat }}\right)$, of 0.068 ( $\pm 0.008,2$ s.e.m.). In contrast, the selective effect of the gat $Z$ mutant was smaller in $\operatorname{Rag} 2^{-1-}$ mice $\left(s_{\text {gat }}=0.03\right.$ ( $\pm 0.01,2$ s.e.m. $)$ ) compared with WT (analysis of variance (ANOVA) with Tukey's post hoc test, $\left.P<10^{-3}\right)$. However, considerably higher variation in the selective effects of the gat $Z$ mutation was found in Rag2 ${ }^{-1}$ mice (Fig. 2c, right panel; Supplementary Table 1), with a two-tailed test for variance in $s_{\text {gat }}$ between $R a g 2^{-/}$and WT being marginally significant $(\mathrm{F}=0.30, P=0.09)$. The mutation shows antagonistic pleiotropy, that is, in some Rag $2^{-1-}$ mice it was advantageous (Rag2 ${ }^{-1}-1$ and 5), while in others it was neutral or slightly deleterious (Rag2 $2^{-/}-4$ and 10$)$. We note that variability for the fitness effect of the mutant was observed in mice from the same litter (for example, Rag $2^{-1}$ - littermates 3 and 4 or 5 and 6 in Fig. 2c). The strength of natural selection is therefore the principal source of variation in the evolutionary dynamics between the hosts. These results suggest that the 


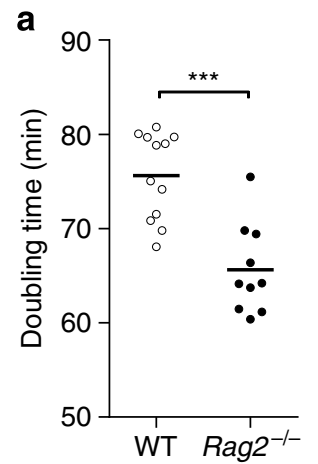

C

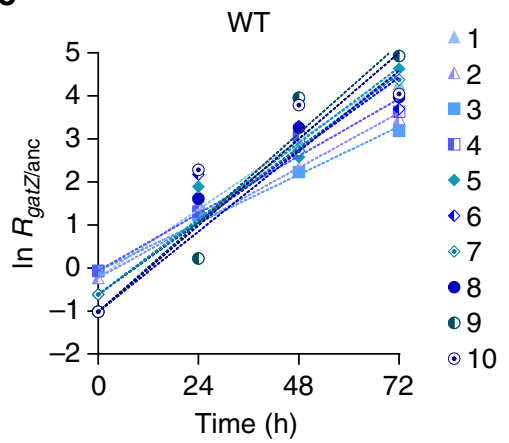

b

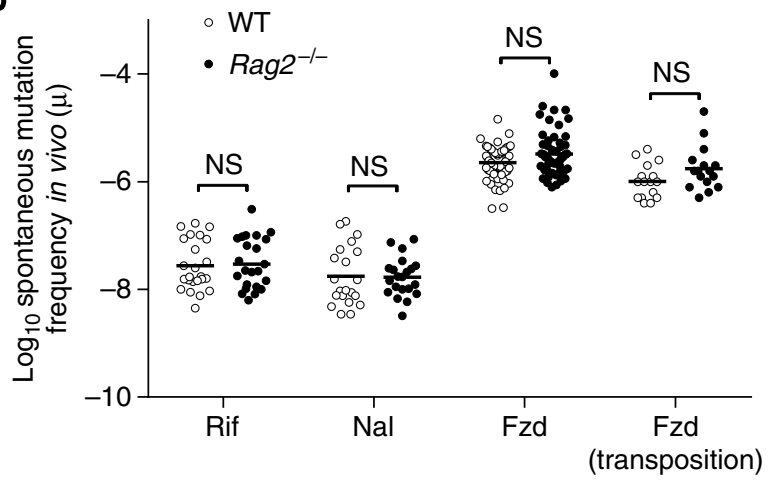

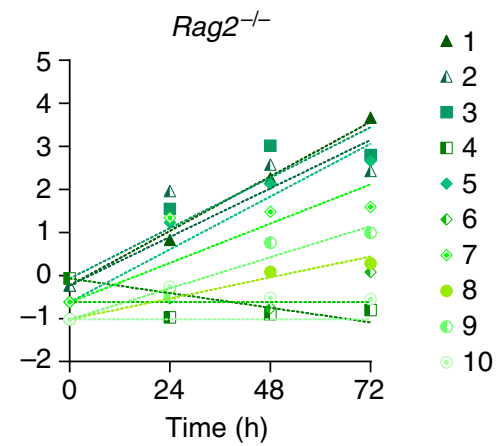

Figure 2 | Selection strength, not mutation rate, is responsible for a lower rate of adaptation in immune-compromised mice. (a) Higher growth rate of E. coli in immune-compromised mice. The doubling time of E. coli colonizing the gut of WT or Rag $2^{-/-}$is estimated by the amount of rRNA (see Supplementary Fig. 3). Each symbol corresponds to an average of two independent quantifications made for each mouse ( $n=6)$ during days 1 and 3 of colonization. The doubling time is significantly lower in Rag2 ${ }^{-/-}$than in WT mice, indicating that $E$. coli divides faster in the gut of Rag2 ${ }^{-/-}$than in WT animals (Mann-Whitney $U$-test, $W=111.5, P<10^{-3}$ ). ${ }^{\star \star \star} P<0.001$. (b) Similar frequency of spontaneous mutation in immune-competent and immunecompromised mice: the $\log _{10}$ frequency of spontaneous resistance $(\mu)$ in WT (open circles) and in Rag2 ${ }^{-/-}$(full circles) animals is proportional to the mutation rate at mutation-selection balance. Symbols correspond to measurements of mutation frequency over a period of 15 days for rifampicin ( $n=6$ ), nalidixic acid $(n=6)$ and furazolidone resistance $\left(n=11\right.$ and 10). We estimated an average $\log _{10} \mu_{\mathrm{WT}} \sim-7.56$ and $\mu_{\text {Rag } 2}^{-1} \sim-7.53$ for rifampicin $($ MannWhitney $U$-test, $W=284.5, P=0.95), \mu_{\mathrm{WT}} \sim-7.76$ and $\mu_{\text {Rag2 }}^{-} \sim-7.77$ for nalidixic acid (Mann-Whitney $U$-test, $W=202, P=0.65$ ) and $\mu_{\mathrm{WT}} \sim-5.65$ and $\mu_{\text {Rag2 }}^{-} \sim-5.49$ for furazolidone resistances (Mann-Whitney $U$-test, $W=1613, P=0.10$ ). The frequency of spontaneous transpositions corresponds to the fraction of mutations conferring furazolidone resistance, which are caused by insertion of transposable elements: $\mu_{\mathrm{TE}} \mathrm{WT}_{\mathrm{T}} \sim-5.99$ and $\mu_{\mathrm{TE}}^{-} / \overline{R a g} 2$ $\sim-5.75$ (Mann-Whitney $U$-test, $W=83, P=0.09$ ). NS, not significant; $P>0.05$. (c) Mean selective advantage of an emerging gat allele is lower in Rag2 ${ }^{-/}$- than in WT mice. Results of short-term competitive fitness experiments $(n=10)$ between a gatZ mutant and the ancestral E. coli. Rag2 $2^{-}-$ (right panel) or WT (left panel) mice from the same litter are represented by symbols of the same shape (triangles, squares, diamonds or circles). The selective advantage of gat $Z$ along the 3 days of competition is inferred from the slope of the linear regression of $\ln ($ gat $Z /$ anc), (dashed lines, shown for each mouse), which corresponds to the selection coefficient.

selective pressures in the gut of immune-compromised and WT mice are different.

Differences in host immune status are associated with differences in microbiota species composition: the microbiota of mice lacking $\mathrm{T}$ and $\mathrm{B}$ cells has been shown to differ from that in WT controls ${ }^{7}$. To investigate the potential role of the microbiota in driving the changes in adaptation rate observed in this study, we estimated $s_{\text {gat }}$ by direct in vivo competition against the ancestral in (i) WT and Rag2 ${ }^{-1-}$ mice previously co-housed for 1 month, a procedure that leads to homogenization of the microbiota between different individuals ${ }^{15}$ (Fig. 3a; Supplementary Table 2), and (ii) germ-free (GF) WT and Rag $2^{-1-}$ mice devoid of microbiota (Fig. 3b; Supplementary Table 3). Analysing the full data set (Fig. 3c) including

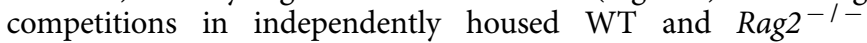
animals, revealed a significant overall interaction between host immune status and the microbiota (ANOVA; $\mathrm{F}_{(2,50)}=9.64$; $P<10^{-3}$ ), upon the fitness effect of the emerging mutation. In WT animals, co-housing slightly $\left(s_{\text {gat }}=0.05 \pm 0.01\right)$ but not significantly (ANOVA with Tukey's post hoc test, $P=0.3$, Fig. 3c) decreased the mutant fitness (Fig. 3a, left panel). This advantage was similar to that measured in co-housed Rag2 ${ }^{-1-}$ $\left(s_{\text {gatZ }}=0.050 \pm 0.009\right.$, ANOVA with Tukey's post hoc test, $P>0.99)$, which was higher than in independently housed Rag2 ${ }^{-1-}$ (ANOVA with Tukey's post hoc test, $P=0.02$ ). Another marked effect of the co-housing protocol was a reduction in the variance for the fitness effect of gat $Z$ in Rag2 ${ }^{-1-}$ mice, which became more similar to that observed in WT animals (F-test, $\mathrm{F}=1.32, \quad P=0.67$ ), indicating loss of antagonistic pleiotropy.

Analysis of GF animals provided further evidence of a major role for the microbiota in shaping the selective pressure on the gatZ mutant. First, $s_{\text {gat }}$ was smaller in GF animals than in microbiota-bearing WT animals (ANOVA with Tukey's post hoc test, $P<10^{-3}$, Fig. 3c), even though a shorter duplication time was found for E. coli in the former (see Supplementary Fig. 4). Second, both the mean and the variance for $s_{\text {gat }}$ were similar between GF WT and Rag2 ${ }^{-1-}$ mice (ANOVA with Tukey's post hoc test, $P>0.99$, Fig. $3 c$; and F-test, $\mathrm{F}=0.97, P=0.97)$. Finally, irrespective of the functional state of the immune system, the variance for $s_{\text {gatz }}$ decreased markedly in GF compared with microbiota-harbouring animals (F-test, $P<10^{-3}$ ). Globally, these 
a
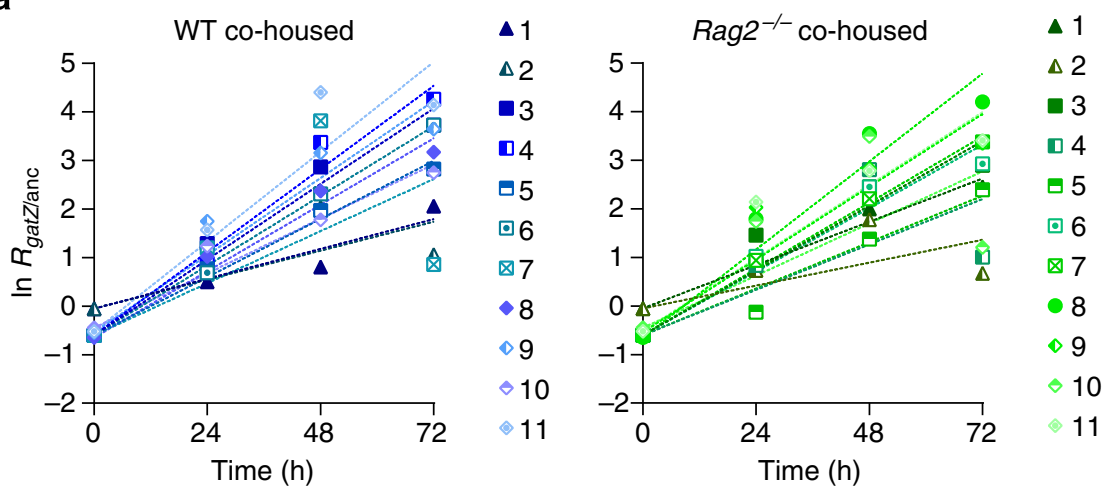

b
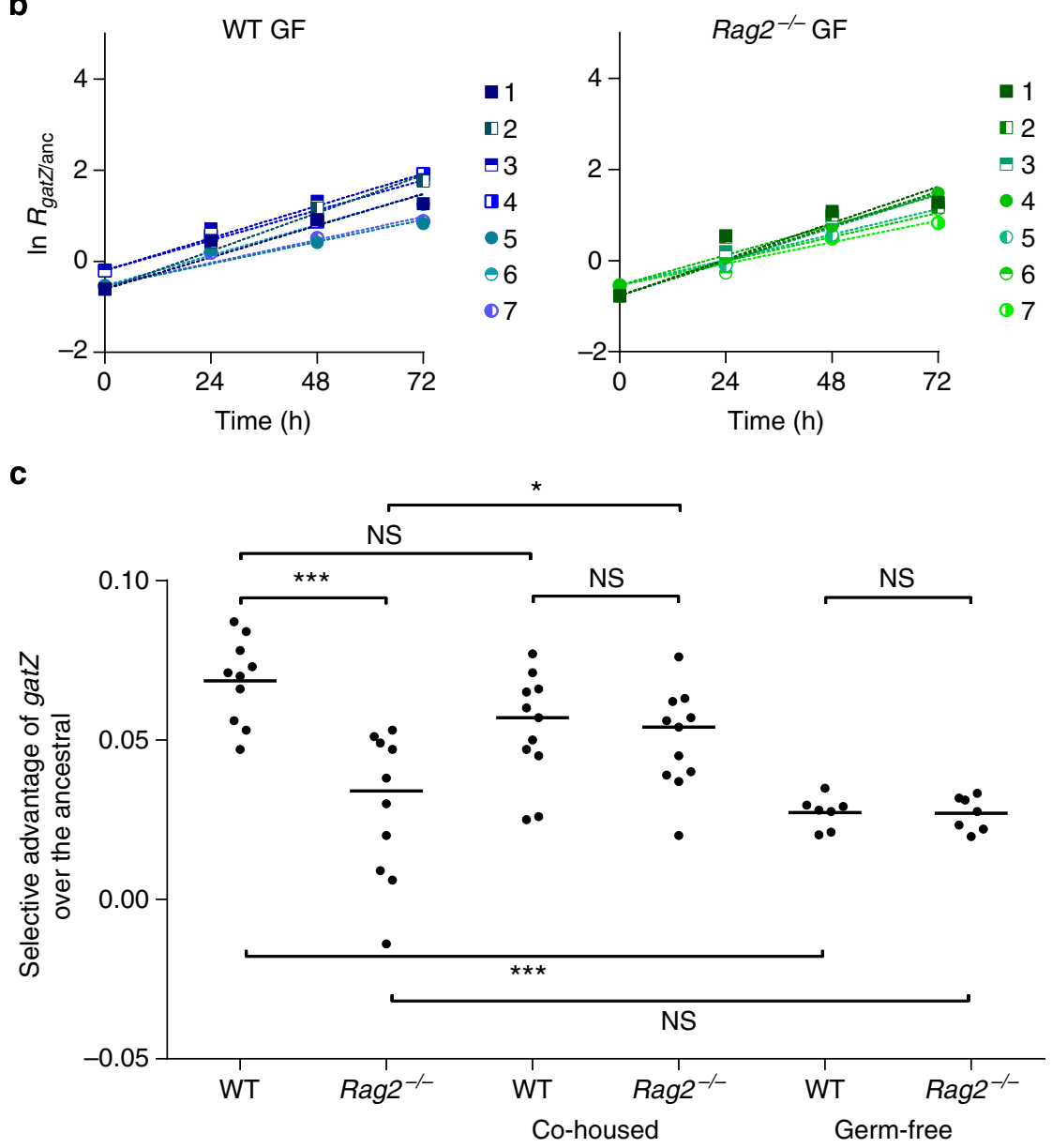

Figure 3 | The microbiota influences the selective advantage of adaptive mutations. Competitive fitness experiments of the emerging gat $Z$ allele against the ancestral strain in (a) WT co-housed with Rag2 ${ }^{-/}$- mice (left panel, $n=11$ ) and Rag2 ${ }^{-/}$- co-housed with WT mice (right panel, $n=11$ ) and in (b) GF

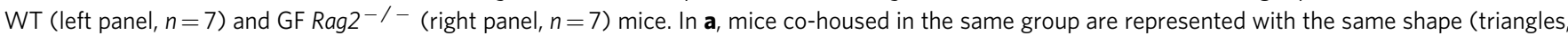
squares or diamonds). Advantage of gatZ over the ancestral, per hour was calculated as in Fig. 2c. (c) Selective advantage of the gatZ mutant, per hour, inferred from the slope of the linear regression of $\ln ($ gatZ/anc), over 3 days of in vivo competition against the ancestral in mice either WT, Rag2- /- , WT

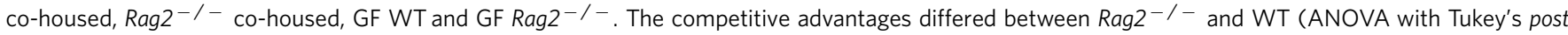
hoc test, $P<10^{-3}$ ), but upon co-housing the advantage in Rag2 ${ }^{-/}$mice (co-housed) increased (ANOVA with Tukey's post hoc test, $P=0.02$ ) and became similar to WT animals (co-housed) (ANOVA with Tukey's post hoc test, $P>0.99$ ). The advantage of the WT did not significantly change upon cohousing (ANOVA with Tukey's post hoc test, $P=0.3$ ). In the absence of microbiota (GF), the selective effect of gat $Z$ is similar in WT (circles) and Rag2 ${ }^{-} /-$ (triangles) mice (ANOVA with Tukey's post hoc test, $P>0.99$ ) and smaller than in WT mice harbouring microbiota (ANOVA with Tukey's post hoc test, $\left.P<10^{-3}\right)$. NS, not significant; $P>0.05,{ }^{\star} P<0.05,{ }^{\star \star \star} P<0.001$.

results confirm the microbiota as a major player modulating the selective effect of beneficial mutations.

When competing two single gat mutants, one carrying an IS insertion in gat $Z$ (previously used for the competitions against the ancestral) and the other a single-nucleotide polymorphism (SNP) in gatC (see Methods), in independently housed (Fig. 4a; Supplementary Table 4) or co-housed (Fig. 4b; Supplementary Table 5) WT and Rag2 $2^{-1}$ mice similar results were obtained. 

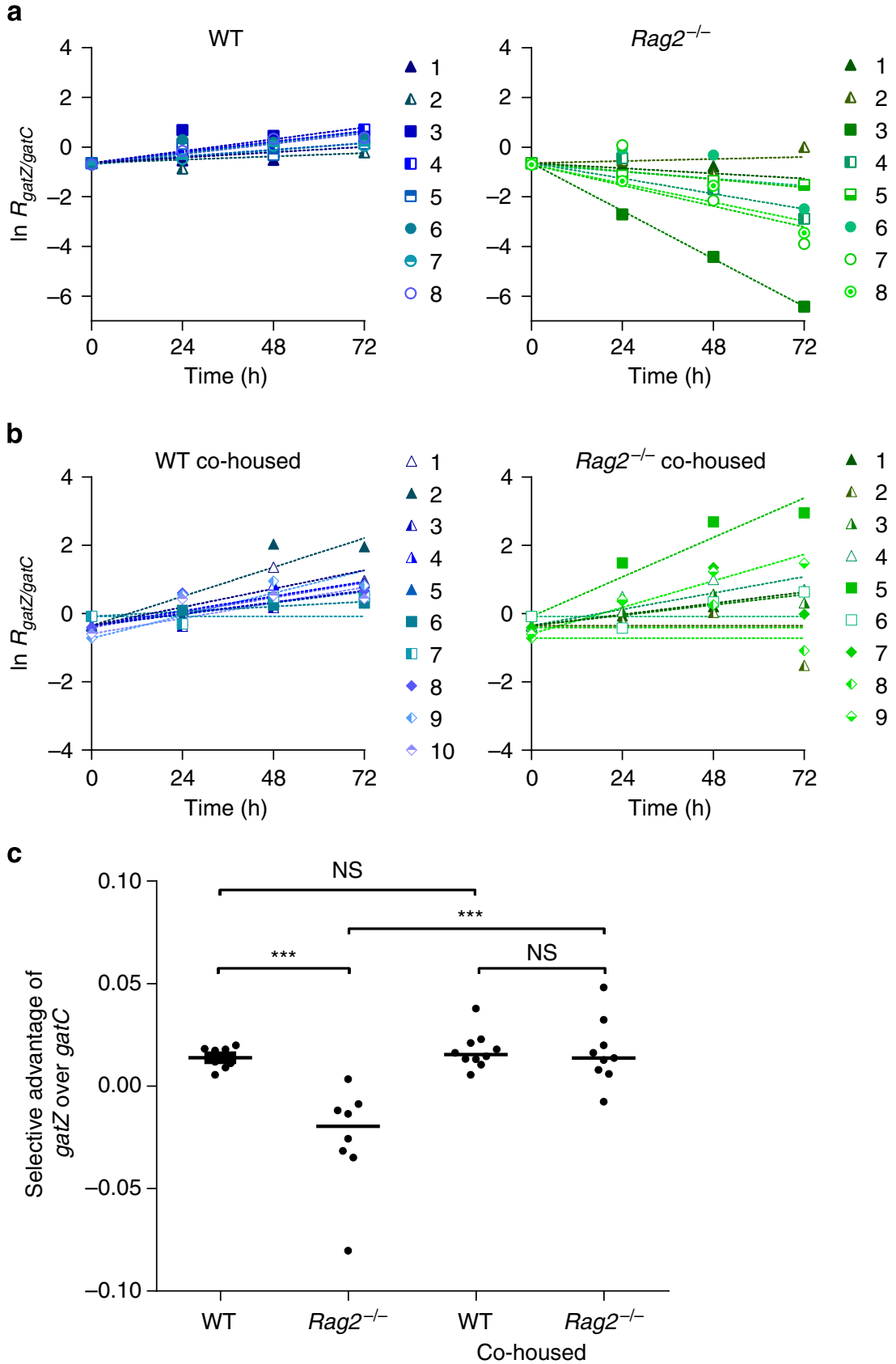

Figure 4 | Evidence for antagonistic pleiotropy in immune-compromised mice. Selective effect of gatZ allele relative to gatC allele in (a) WT (left panel) and Rag2 ${ }^{-1-}$ (right panel), (b) WT co-housed with Rag2 ${ }^{-/-}$mice (left panel) and Rag2 ${ }^{-/}$- co-housed with WT mice (right panel). Competitions were performed in independently housed WT and Rag2 ${ }^{-/-}$mice $(n=8)$ or in mice co-housed for 1 month $(n=9)$. In $\mathbf{a}$, mice from the same litter are represented by the same shape symbols (triangles, squares or circles) and in $\mathbf{b}$, mice co-housed in the same group are represented with the same shape (triangles, squares or diamonds). (c) Comparison of the selective effects of the gat $Z$ over gatC inferred from the slope of the linear regression of $\ln (\mathrm{gat} Z / \mathrm{gat} C$ ), over 3 days of

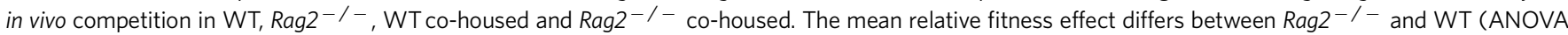
with Tukey's post hoc test, $P<10^{-3}$ ), but upon co-housing no significant difference was detected between animals (ANOVA with Tukey's post hoc test $P>0.99$ ). Co-housing with WT results in an increase in the mean selective effect of gat $Z$ versus gat $C$ in immune-compromised mice (ANOVA with Tukey's post hoc test, $P<10^{-3}$ ) but not in WT mice (ANOVA with Tukey's post hoc test, $P>0.99$ ). NS, not significant, $P>0.05,{ }^{\star \star \star} P<0.001$.

Again, we found a significant interaction between the microbiota and the presence of an adaptive immune system (ANOVA; $\mathrm{F}_{(2,31)}=12.9 ; P=0.001$ ). As expected, in WT mice (Fig. 4a, left panel) the mutants were almost neutral $\left(s_{\text {gatZvsgat } C}=\right.$ $0.01 \pm 0.004)$. However, in Rag $2^{-l-}$ mice (Fig. 4a, right panel) $s_{\text {gatZvsgat }}$ was on average smaller $(-0.03 \pm 0.02$, ANOVA with Tukey's post hoc test, $P<10^{-3}$, Fig. 4c). Extensive variability for the fitness effects was observed, with an extreme case in which gat $Z$ was found to have a strongly deleterious effect (Fig. 4a, Rag $2^{-1-}$ mouse 3). On the other hand, when performing the same competitions in co-housed animals (Fig. 4b), the average $s_{\text {gatZvsgatC }}$ in Rag $2^{-I-}$ mice increased (ANOVA with Tukey's post hoc test, $P<10^{-3}$, Fig. 4c), approximating the value observed in co-housed WT $\left(s_{\text {gatZvsgatC }}=0.02 \pm 0.01\right.$, ANOVA with Tukey's post hoc test $P>0.99$, Fig. 4c). Finally, we also detected a significant increase in 
the variance for $s_{\text {gat }}$ when competed against gat $C$ in independently housed $R a g 2^{-I-}$ compared with WT animals (F-test, $\mathrm{F}=0.04, P<10^{-3}$ ).

Our combined fitness data provide strong evidence for antagonistic pleiotropy specific to immune-compromised animals, a feature that reduces the predictability of E. coli evolution in these hosts. Moreover, our results indicate that the host, the microbiota and their interactions influence the pace of adaptive evolution of E. coli to the mouse gut. Together, these findings suggest that a microbiota shaped by the adaptive immune system provides stronger selective pressures upon an individual bacterial species residing within this environment, which arises in the absence of this host component.

Microbiota characterization of WT and $\operatorname{Rag}^{-/-}$mice. Recent studies have reported differences in microbiota composition between immune-competent and immune-compromised animals ${ }^{6,7,16-20}$. To determine whether changes in the microbiota within WT and $R a g 2^{-1-}$ raised in our facility were associated with the slower rate and increased variation observed in $E$. coli adaptation in immune-compromised animals, we assessed the composition of this community following high-throughput $16 \mathrm{~S}$ rRNA gene sequencing of DNA extracted from faecal samples of unmanipulated animals (Supplementary Fig. 5) and from day 3 of the evolution experiment (Fig. 5).

In unmanipulated hosts $(n=5$ for each, see Microbiota analysis in the Methods section), the phylogenetic diversity was significantly higher in WT when compared with Rag2 ${ }^{-1-}$ animals (MannWhitney $U$-test, $W=23.5, P=0.03$, Supplementary Fig. 5a). Consistent with this result, PCoA analysis on the phylogeny-based distance of community membership showed a significant difference between genotypes (analysis of molecular variance (AMOVA) on unweighted UniFrac distance, $P=0.003$, Supplementary Fig. 5b). However, at the genus level, difference in abundance was only observed for some low-frequency genera, albeit not statistically significant (Supplementary Fig. 5c). Similarly, operational taxonomic unit (out)-based measures of community richness and diversity revealed only a trend, not significant, for decreased community diversity in Rag2 ${ }^{-1-}$ (Mann-Whitney U-test, $W=17, P=0.4$ and $W=21, P=0.1$, respectively, Supplementary Fig. 5d,e). PCoA analysis of OTU- and phylogeny-based distances of community structure revealed no significant differences (AMOVA on Bray-Curtis distance, $P=0.1$ and weighted UniFrac distance, $P=0.4$, Supplementary Fig. 5e,f). Importantly, reads identified as Escherichia/Shigella could be detected at very small and similar frequencies (maximum $0.03 \%$ ) in both Rag2 ${ }^{-1-}$ and WT animals (Mann-Whitney $U$-test, $W=8.5, P=0.46$ ), suggesting that direct competition between our experimental E. coli and native Escherichia, if any, would be similar in both hosts.

We then analysed $10 \mathrm{WT}$ and $10 \mathrm{Rag} 2^{-1-}$ animals from day 3 of the evolution experiment, that is, treated with streptomycin and colonized with $E$. coli. The gut microbiota was dominated at the genus level by a few bacterial groups (Fig. 5a), compatible with the strong effect of this treatment, as shown before ${ }^{21}$. In this setting, we identified genera differentially represented in WT and Rag2 ${ }^{-1}-$ mice (Fig. 5b). Most notably, Barnesiella, Allobaculum and an unclassified genus of Clostridiales were more abundant in Rag2 ${ }^{-1}-$ mice, while Bacteroides, and Paenibacillus were more prevalent in WT animals. In line with the variance observed when monitoring the gat mutant fitness, we detected increased variance in Rag2 ${ }^{-T-}$ mice for these genera, particularly that of Barnesiella (F-test, $\mathrm{F}=16.3, P<10^{-3}$ ), for which the relative frequencies ranged between 2 and 74\%, while in WT hosts the range was from 0 to $17 \%$. In mice where the rate of increase of the gat-negative phenotype (as a proxy for rate of adaptation) was the highest (R1.10) or the lowest (R1.11) (see Supplementary Fig. 2), the microbiota was composed of a small (2\%) or large (51\%) frequency of Barnesiella, respectively.

Although the richness of microbial communities was similar between streptomycin-treated WT and Rag2-/- animals (Mann-Whitney $U$-test, $W=39, P=0.4$, Fig. 5c), both OTUbased and phylogenetic diversity were increased in the latter (Mann-Whitney $U$-test, $W=20, P=0.03$ and $W=17, P=0.01$, Fig. 5d,e). Strikingly, when comparing the beta diversity metrics of treated WT and Rag2 $2^{-1-}$ animals, PCoA analysis of BrayCurtis (OTU-based) and weighted UniFrac (phylogeny-based) distances revealed that the structure of microbial communities significantly differed between the two genotypes (AMOVA on Bray-Curtis distance, $P=0.01$ and weighted UniFrac distance, $P=0.03$, Fig. 5f,g). However, only marginally significant differences were found for the phylogenetic membership (AMOVA on the unweighted UniFrac distance, $P=0.08$, Supplementary Fig. 6).

These results indicate that the microbiota ecosystem experienced by $E$. coli in our experimental setting differs between WT and Rag $2^{-1-}$ hosts and between Rag2 $2^{-1-}$ individuals, a feature strikingly reminiscent of the gat mutant fitness.

Genetic targets of adaptation in WT and $\operatorname{Rag}^{-/-}$mice. We and others have previously shown that over a 3-week period of evolution, more than one genetic target can be involved in the genetics of $E$. coli adaptation to the mouse gut $^{11,22,23}$. We therefore thought of determining whether the haplotypic

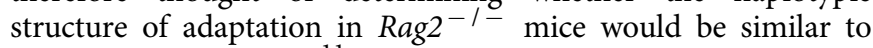
that of WT animals ${ }^{11}$. We sampled clones $(\sim 20)$ from independent E. coli populations recovered from each Rag2 $2^{-1-}$ mouse after 24 days of evolution. Clones were typed for the presence of mutations previously found to be under selection in the gut of WT mice ${ }^{11}$, namely: IS insertions in the intergenic regions of $f o c A / y c a O$ (formate transporter), $d c u B / d c u R$ (fumarate transporter) and SNPs in $s r l R$ (regulator of the sorbitol metabolism). In Rag $2^{-1-}$ animals (Fig. 6), IS insertions near focA/ycaO as well as SNPs in the srlR gene were common (found in 10 and 9 out of 15 mice, respectively). These mutations were segregating at different frequencies within each host: $10-90 \%$ for focA $/ y c a O$ and $10-100 \%$ for $s r l R$ mutations. Strikingly, insertions near $d c u B / d c u R$ were not found in the E. coli that had evolved in immune-deficient animals (Fig. 6a), although this target was repeatedly identified in clones sampled from WT mice ${ }^{11}$.

The clear occurrence of clonal interference-where clones carrying distinct haplotypes competing for fixation within a population-among E. coli evolving in both Rag2-/- (Fig. 6) and WT mice ${ }^{11}$, together with the similar rate of spontaneous transposition estimated in these hosts (Fig. 2b), suggests that mutation is non-limiting in the gut of both animals. In turn, this property argues for insertions near $d c u B$ to be beneficial in WT, but neutral or even slightly deleterious in $R a g 2^{-1-}$. To test this hypothesis and identify other targets of adaptation, we performed whole-genome sequencing (WGS) of large population samples ( $>1,000$ clones) that had evolved in each animal. This approach allows the identification of mutations segregating at high frequency, and thus likely to be beneficial, but not those present at low frequency. As expected, WGS (Table 1; Supplementary Tables 6 and 7) revealed the gat operon, the $\operatorname{srl} R$ locus and the intergenic region of $f o c A / y c a O$ as the main targets of adaptation in both hosts. Remarkably, $d c u B$ was hit in $7 / 14 \mathrm{WT}$ and in none of the $15 R a g 2^{-1-}$ population tested (binomial test, $P=10^{-3}$ ), confirming the beneficial effects of insertions near this gene in immune-sufficient and not in immune-deficient mice.

The WGS approach also revealed new bona fide targets for E. coli adaptation to the mouse gut (Table 1). These are targets 

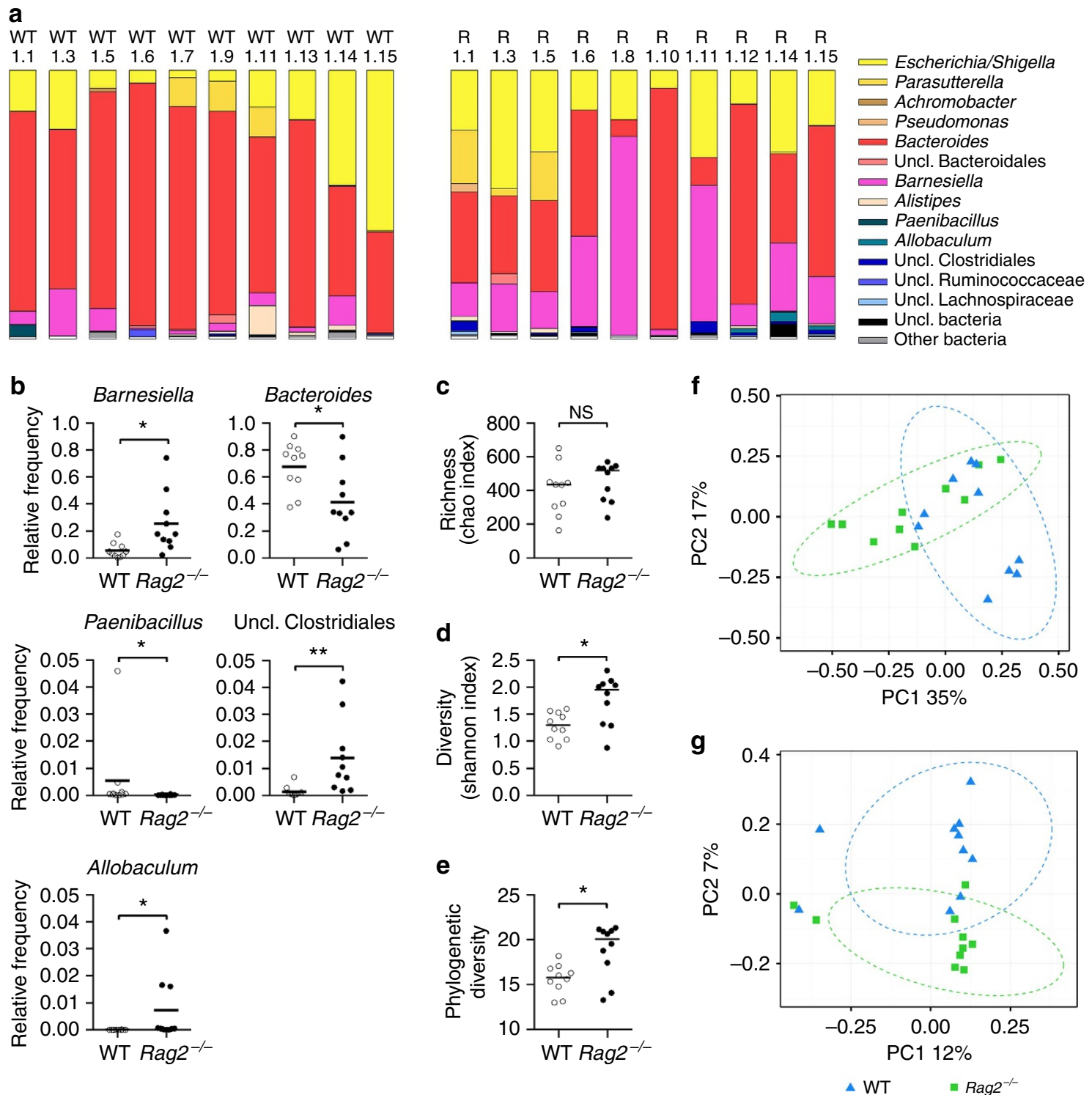

Figure 5 | Microbiota composition differs between immune-competent and immune-compromised hosts. (a) Microbiota composition at the genus level of $10 \mathrm{WT}$ and $10 \mathrm{Rag}^{-1}$ - mice from the day 3 of the evolution experiment (streptomycin-treated and colonized with E. coli). Genera with a relative abundance larger than $1 \%$ are displayed. The coloured segments represent the relative frequency of each genus. (b) Relative frequencies of genera differentially represented in WT and Rag2 ${ }^{-1-}$ animals: Barnesiella (Mann-Whitney U-test with the Benjamini and Hochberg correction, $W=11, P=0.021$ ), Bacteroides (Mann-Whitney $U$-test with the Benjamini and Hochberg correction, $W=18, P=0.049$ ), Paenibacillus (Mann-Whitney $U$-test with the Benjamini and Hochberg correction, $W=14.5, P=0.029$ ), unclassified Clostridiales (Mann-Whitney U-test with the Benjamini and Hochberg correction, $W=6, P=0.0065$ ) and Allobaculum (Mann-Whitney U-test with the Benjamini and Hochberg correction, $W=13, P=0.025)$. ${ }^{\star} P<0.05,{ }^{\star \star} P<0.01$. Alpha diversity estimates of microbiota community richness (c), diversity (d) and phylogenetic diversity (e) in WT and Rag2 ${ }^{-1}-$ animals. Both OTU-based diversity and phylogenetic diversity are increased in Rag2 ${ }^{-} /$- animals compared with WT (Mann-Whitney $U$-test, $W=20, P=0.03$ and $W=17, P=0.01$, respectively). NS, not significant; $P>0.05$, ${ }^{\star} P<0.05$. Principal coordinate analysis ( $\left.P C o A\right)$ of the Bray-Curtis (f) and weighted UniFrac ( $(\mathbf{g})$ distance matrices of faecal microbiota of WT and Rag $2^{-/-}$mice. The first two coordinates are shown. Ellipses centred on the averages of the metric distances with a $90 \%$ confidence interval for the first two coordinates were drawn on the associated PCoA.

that occurred in at least two E. coli populations evolving in mice living in allopatry. We detected IS insertions, deletions and nonsense mutations in the coding region of $k d g R$, a transcriptional repressor ${ }^{24}$ regulating the metabolism of sugar acids ${ }^{25}$, and insertions of IS elements in the intergenic region of $y j j P$ and $y j j Q$, that code, respectively, for an predicted inner membrane protein and a putative transcription factor ${ }^{26}$. We also found an additional target specific to WT mice: yeaR, encoding a protein induced in the presence of nitric compounds 27 .

Interestingly, we identified parallel mutational targets detected only in immune-compromised mice ( $\operatorname{arc} B$, frlR and $\operatorname{rim} J)$. $\operatorname{arcB}$ encodes a transmembrane sensor kinase and together with $\operatorname{arcA}$, regulates the expression of the Arc modulon in response to changes in oxygen levels ${ }^{28}$. frlR codes for a predicted regulator of the fructoselysine operon ${ }^{29}$ that is responsible for the metabolism of fructosamines. rimJ encodes an alanine acetyltransferase involved in modification of ribosomal proteins ${ }^{30}$.

\section{Discussion}

We investigated the process of $E$. coli evolution in the gut of immune-compromised mice and compared it with the 
$\mathrm{R} 1.1$
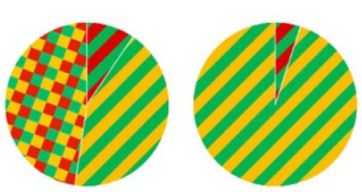

R1.9
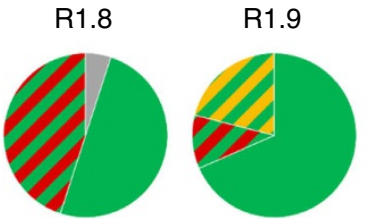

R1.3

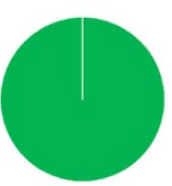

R1.10

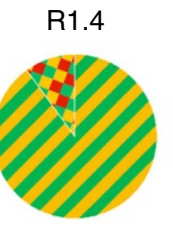

R1.11

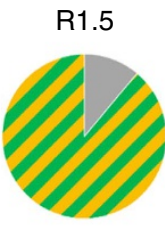

$\mathrm{R} 1.12$

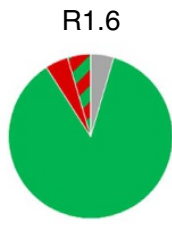

R1.13
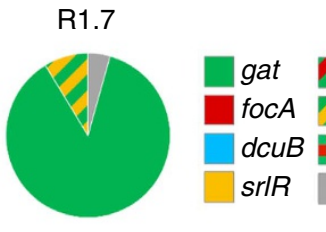

gat focA

gat srIR

cuB gat focA srlR

srlR No mutation
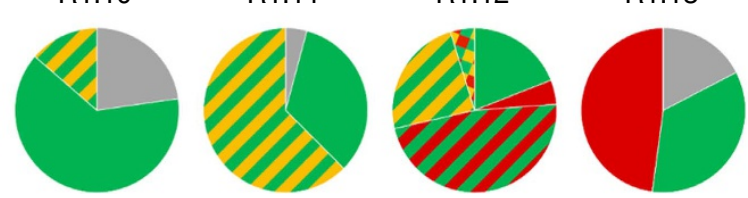

R1.14

R1.15
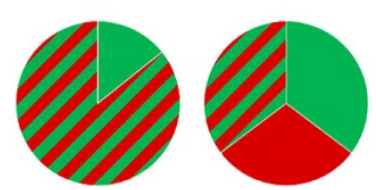

Figure 6 | Host genetics influences the genetic basis of $\boldsymbol{E}$. coli adaptation in the gut. Frequencies of haplotypes in E. coli populations evolved in the gut of Rag $2^{-/}$mice for 24 days. Clones $(n \sim 20)$ isolated from the last day of evolution of lineages R1.1 to R1.15 (Fig. 1b) were screened for the presence of mutations previously identified in WT animals: gat phenotype (green), insertions in the regulatory region of focA (red) and dcuB (blue) and SNPs in the coding region of srlR (orange). Clones in which none of these mutations were found are shown in grey. Clonal interference is detected by the competition between clones carrying different beneficial mutations (stripes of different colours represent double mutants and clones carrying three mutations are shown as a checkered pattern).

\begin{tabular}{|c|c|c|c|}
\hline \multirow[t]{2}{*}{ Gene } & \multirow[t]{2}{*}{ Function } & \multicolumn{2}{|c|}{ Frequency of mice } \\
\hline & & WT & $\operatorname{Rag2}^{-/-}$ \\
\hline gat Operon & Metabolism of galactitol & $100 \%( \pm 7 \%)$ & $100 \%( \pm 7 \%)$ \\
\hline srlR & Metabolism of sorbitol & $50 \%( \pm 13 \%)$ & $60 \%( \pm 13 \%)$ \\
\hline focA $/ y c a O$ & Anaerobic respiration (formate transporter) & $29 \%( \pm 12 \%)$ & $40 \%( \pm 13 \%)$ \\
\hline yjjP/yjjQ & Inner membrane protein & $57 \%( \pm 13 \%)$ & $13 \%( \pm 9 \%)^{\star \star \star}$ \\
\hline yeaR & Metabolism of nitric compounds & $14 \%( \pm 9 \%)$ & $0 \%$ \\
\hline $\operatorname{arc} B$ & Regulation of respiration & $0 \%$ & $13 \%( \pm 9 \%)$ \\
\hline frlR & Metabolism of fructosamines & $0 \%$ & $13 \%( \pm 9 \%)$ \\
\hline rim & Modification of ribosomal proteins & $0 \%$ & $13 \%( \pm 9 \%)$ \\
\hline
\end{tabular}

WGS, whole-genome sequencing; WT, wild type.

Frequency ( \pm 2 s.e.m.) of WT $(n=14)$ or Rag $2^{-/-}(n=15)$ populations in which parallel mutations at a given locus were found segregating at high frequency ( $\left.>10 \%\right)$. These loci, identified through WGS of population samples, were observed in at least two populations and thus correspond to bona fide targets of beneficial mutations. Mutations whose emergence differs significantly between the two WGS of population samples, were observed in at least two populations and thus correspond to bona fide targets of benef
host genetic backgrounds are highlighted in bold, with the level of significance displayed (binomial test $* \star *<<0.001$ ).

evolutionary patterns observed for the same ancestral strain in WT mice. We observed a difference in the initial divergence of the neutral marker in immune-compromised compared with immune-competent animals, compatible with a slower rate of evolution in the former. This finding is further supported by a delayed emergence of the adaptive gat phenotype, whose dynamics displayed a slower pace and increased variability in its fitness effects among these hosts. In a system of intense clonal interference, as is the case here, the power of the two-marker system in detecting adaptive mutations becomes rapidly reduced. Consequently, the lack of differences in marker dynamics between hosts over longer periods is not interpretable.

Our findings of a higher growth rate of E. coli in Rag2-/animals compared with WT is compatible with a weakened control of bacterial growth in immune-compromised hosts, suggesting a more benign environment for E. coli. The same explanation may also be valid for the GF mice, where even lower duplications times, regardless of the immune state, were observed. In agreement with this conjecture, the selective effects of the first sweeping beneficial mutations were smaller in immune-compromised hosts, indicating stronger selective pressures in WT mice.

The bulk of our work indicates that the adaptive immune system indirectly increases this selective pressure through the shaping of the microbiota, which in turn interacts with E. coli.
First, alteration (co-housing) or removal (GF) of the microbiota modulates the selective effects, clearly revealing that this is a major factor controlling the selective pressures in the mouse gut. Second, we could indeed detect differences in the relative abundance of some genera, notably Barnesiella spp, between the two host genotypes. Bacteria belonging to this genus have been shown to confer protection against vancomycin-resistant Enterococcus, through some undefined direct or indirect mechanism ${ }^{31}$. Our results obtained upon antibiotic treatment in $R a g 2^{-1-}$ animals, not only show an increase in the mean frequency of Barnesiella but also a substantial degree of inter-individual variation, supporting a role for the host adaptive immune system in maintaining coherence in the ecology and evolution of the commensal microbes.

The influence of the host immune system, in particular the adaptive immune system, on the shaping of the gut microbiota has been the focus of recent studies. Among these, the microbiota composition of WT and Rag1 ${ }^{-1-}$ or Rag2 ${ }^{-1-}$ mice (both Rag1 and Rag2 null mutations result in total ablation of the adaptive immune system) has been compared using $16 \mathrm{~S}$ analysis $7,16,17,19$. While each study reports differences in the representation of several microbial groups, these are not the same across animal facilities. For example, one work revealed an expansion of anaerobes, especially SFB, in the gut of Rag2 $2^{-1-}$ mice $^{18}$, while 
others described differences in the abundance of Bifidobacteria and Clostridium leptum ${ }^{19}$, or in the phylogenetic community membership such as increased Lachnospiraceae and decreased Porphyromonadacea in $\mathrm{Ragl}^{-1-}$ mice $^{1 t}$. None of these specific differences were noticeable in our colonies. In addition, a previous study reported an increased frequency of Akkermansia sp in Rag2 ${ }^{-1}$ - hosts when compared with WT controls ${ }^{7}$. While this genus was represented at notable frequency (5\%) in one unmanipulated $R a g 2^{-1-}$ animal in our study, it was undetectable in the streptomycin-treated mice analysed here, irrespectively of their genotype. However, we detected differences in phylogenetic memberships (unweighted UniFrac) between unmanipulated WT and Rag2 ${ }^{-I-}$ animals, confirming that the two genotypes do carry different microbial gut compositions. Moreover, our observation of reduced phylogenetic diversity in unmanipulated $\mathrm{Rag}^{-1-}$ corroborates other works, where immune-compromised mice, including mutants lacking either or both $\mathrm{T}$ and $\mathrm{B}$ cells display decreased microbial diversity ${ }^{6}$. A similar finding was reported recently, where the abundance and diversity of B-cell-secreted immunoglobulin-A was correlated with the diversity of the gut microbiota ${ }^{32}$.

More directly relevant to the scope of this work, we found differences in both phylogeny- and OTU-based measures of alpha diversity between streptomycin-treated WT and Rag2 ${ }^{-1-}$ mice. Together with our findings of distinct community structures (weighted UniFrac and Bray-Curtis distances) between the two genotypes, these results suggest that the pre-existing differences in microbial composition between WT and $\mathrm{Rag}^{-{ }^{-}}$are exacerbated upon antibiotic treatment. Overall, and in agreement with the majority of previous works, our results indicate that microbial composition differs between immune-competent and -compromised animals, supporting the hypothesis that adaptive immunity plays an important role in the shaping of the microbiota.

The genetic basis of E. coli adaptation determined by WGS of evolved populations revealed that most of the mutations were related with bacterial metabolism and respiration, indicating that the main selective pressure was adaptation to the metabolic environment of the intestine. As the microbiota provides key metabolites $^{33}$ and given our findings of different microbial profiles in WT and Rag2 ${ }^{-1-}$ mice, it is possible that the gut environment of these hosts differs in the availability of different metabolites. In turn, mutations found exclusively or mainly in one host may reflect the specific metabolic composition of that environment. One example, and the most extreme case, is the mutational target $d c u B$ found in half of the WT populations but absent in Rag2 ${ }^{-1-}$ mice. This gene codes for a transporter of C4-dicarboxylic acids such as fumarate and succinate ${ }^{34}$. In the mouse gut, succinate can be produced by Bacteroides ${ }^{35}$, a genus that our microbiota analysis revealed to be present at higher relative abundance in WT than in Rag2 ${ }^{-1-}$ mice. It is possible that differences in the availability of succinate, produced by the microbiota, may have driven the differential selection for mutations in genes related to the uptake and metabolism of this compound in the two groups. The same may be true to yeaR, which codes for a protein induced in the presence of nitric compounds ${ }^{27}$, that can be used by $E$. coli to perform anaerobic respiration in the intestine of the host ${ }^{36}$. Similarly, mutations in frlR, found in Rag $2^{-1-}$ mice, may be related to the levels of fructosamines, compounds that are present in the gut and likely metabolized by bacteria ${ }^{29}$. The mutations identified may lead to inactivation of $f r l R$ and consequently, to the constitutive expression of the $f r l$ operon, conferring an advantage for $E$. coli in the gut when nutrients are available at low concentration. Another example is $k d g R$, a transcriptional repressor of the Entner-Doudoroff aldolase-coding eda gene ${ }^{24}$. Eda is a key enzyme in the Entner-Doudoroff pathway ${ }^{25}$ responsible for the metabolism of sugar acids, and mutants of this pathway are known to display impaired growth in the mouse gut ${ }^{22,37,38}$, which is consistent with our finding of selection for mutations inactivating $k d g R$.

Overall, our data reveal that the path and rate of adaptation of commensal bacteria is influenced by the presence of a healthy adaptive immune system, providing experimental evidence to the first corollary of the co-evolution hypothesis between commensal microbiota and the host immune system. Our findings further suggest that the shaping of the gut microbiota composition by the adaptive immune system results in a coherent environment under which E. coli evolves along a stronger selective pressure and a more predictable path.

\section{Methods}

E. coli and mouse strains. All bacterial strains used in this study were derived from Escherichia coli K-12, strain MG1655 (ref. 39). Strains DM08-YFP and DM09-CFP (MG1655, galK::YFP/CFP ampicillin resistant, $\operatorname{amp}^{\mathrm{R}}(p Z 12)$, streptomycin resistant, $\operatorname{str}^{\mathrm{R}}$ (rpsl150), AlacIZYA), described elsewhere ${ }^{11}$ were used in the colonization experiments. For in vivo fitness assays (see below) two previously described ${ }^{11}$ gat mutants of $E$. coli were used: one harbouring an IS in the coding region of gat $Z$ (clone 4YFP) and another carrying an insertion of $1 \mathrm{bp}$ in gatC (derived from clone 12YFP). These clones were isolated after 24 days of evolution in the WT mouse gut and differed from the ancestral by a single mutation (as confirmed by WGS). Both clones display the gat-negative phenotype.

$\mathrm{C} 57 \mathrm{BL} / 6(\mathrm{WT})$ and $\mathrm{C} 57 \mathrm{BL} / 6 \mathrm{Rag} 2^{-1-}$ mice $\left(\operatorname{Rag}^{-I^{-}}\right)$were used as a model of immune-competent and immune-compromised hosts, respectively. Rag2 ${ }^{-1-}$ animals carry a null mutation in the rag gene essential for the immunoglobulin and $\mathrm{T}$-cell receptor gene recombination and thus lack mature $\mathrm{T}$ and $\mathrm{B}$ lymphocytes. Six-to-eight-week-old WT and Rag2 $2^{-1-}$ male mice bred at the IGC rodent facility and maintained under strict specific pathogen-free (SPF) conditions (barrier facility with autoclaved caging, food and water) were used for all the experiments, unless otherwise stated. For co-housing experiments, 1-month-old WT and Rag2 ${ }^{-1}$ mice (seven animals each) were placed in the same cage for 4 weeks, before $E$. coli colonization. GF C57BL/6 (WT) and C57BL/6 Rag2 ${ }^{-}\left(\right.$Rag2 $\left.^{-1-}\right)$ animals were bred and raised at the IGC gnotobiology facility in dedicated axenic isolators (La Calhene/ORM), an activity partially sponsored by the EU-FP7 InfrafrontierI3EMMA consortium. Young adults were transferred into sterile ISOcages (Tecniplast) before the competition experiments. All animals were kept in individual cages during both the evolution and the competition experiments.

Dynamics of adaptation of $\mathbf{E}$. coli in the mouse gut. To study E. coli adaptation to the gut, we used a streptomycin-treated mouse colonization model ${ }^{40}$. Briefly, SPF-raised mice (see E. coli and mouse strains above) were given autoclaved drinking water containing streptomycin $\left(5 \mathrm{gl}^{-1}\right)$ for 1 day. After $4 \mathrm{~h}$ of starvation for water and food, the animals were gavaged with $100 \mu \mathrm{l}$ of a suspension of $10^{8}$ colony-forming units of a mixture of YFP- and CFP-labelled bacteria (ratio 1:1) grown at $37^{\circ} \mathrm{C}$ in brain heart infusion medium to optical density (OD) $)_{600}$ of 2 . After gavage, the animals were housed in individual cages, and both food and water containing streptomycin were returned to them. Faecal pellets were collected for 24 days, diluted in PBS and plated in Luria Broth (LB) agar. Plates were incubated overnight and the frequencies of CFP- or YFP-labelled bacteria were assessed by counting the fluorescent colonies with the help of a fluorescent stereoscope (SteREO Lumar, Carl Zeiss). A sample of each collected faecal pellet was daily stored in $15 \%$ glycerol at $-80^{\circ} \mathrm{C}$ for further experiments.

For the evolution experiment, groups of five WT and 5 Rag $2^{-1-}$ mice were gavaged and followed for 24 days. This procedure was repeated three times, such that a total of 15 mice of each genotype were analysed. While the results of $E$. col evolution in WT mice were published earlier ${ }^{11}$, experiments in both genotypes were conducted at the same time.

Dynamics of gat-negative phenotype. To investigate the dynamics of appearance and expansion of beneficial mutations in the gat operon, we determined the frequency of bacteria unable to metabolize galactitol (gat-negative phenotype) within a given bacterial population of the evolution experiment ${ }^{11}$. We plated frozen faecal pellets in Mac Conkey agar supplemented with $1 \%$ galactitol and streptomycin $\left(100 \mu \mathrm{g} \mathrm{ml}^{-1}\right)$. Plates were incubated for $20 \mathrm{~h}$ at $28^{\circ} \mathrm{C}$. The frequency of galactitolmetabolizing mutants for each time point was estimated by counting the number of white (gat mutants) and red colonies in Mac Conkey-galactitol plates.

Division rate of $\boldsymbol{E}$. coli in the mouse gut. To determine the growth rate of $E$. coli in the mouse gut, we used a modified version of a previously described method ${ }^{13}$ that takes advantage of the correlation reported to occur between growth rate and ribosomal content of bacterial cells. First, we established a calibration curve (see below) that relates the growth rate of $E$. coli populations growing in a given medium and the fluorescent intensity of a probe specific to E. coli $23 \mathrm{~S}$ rRNA (as a 
measure of ribosomal content). This calibration curve was used to determine the ribosomal content of E. coli cells recovered from faecal pellets of WT and Rag2 ${ }^{-1-}$ mice and infer their growth rates to establish if any differences in the division time of $E$. coli exist in the different hosts.

To obtain a range of growth rates for E. coli, we grew E. coli strain DM09 (ancestral) in M9 minimal medium supplemented with different carbon sources, which support different growth rates. For the initial inoculum, DM09 was grown for $24 \mathrm{~h}$ in $5 \mathrm{ml}$ of $\mathrm{LB}$ supplemented with streptomycin at $37^{\circ} \mathrm{C}$ with aeration. The culture was washed three times in M9 with no carbon source and diluted to OD 2. $2 \mu \mathrm{l}$ of a $10^{-1}$ dilution ( $\sim 10^{5}$ cells) were used to inoculate $198 \mu \mathrm{l}$ of M9 minimal medium supplemented with one of the following carbon sources: ribose $0.4 \%$, sorbitol $0.4 \%$, xylose $0.4 \%$, fructose $0.4 \%$, maltose $0.4 \%$, arabinose $0.4 \%$, gluconate $0.5 \%$ or gluconate $1 \%$. The growth curve assays were performed in a Bioscreen plate incubated in Bioscreen C Microbiology Reader equipment at $37^{\circ} \mathrm{C}$ with continuous shaking. ODs at $600 \mathrm{~nm}$ were monitored for $48 \mathrm{~h}$. All growth measurements were repeated four times. Specific growth rates were calculated based on the slope of the linear regression of the OD increase over time, during exponential growth. This parameter was then used to calculate the growth rates of E. coli in the different media. We then grew new cultures in the same conditions as described above, harvesting the cells in the mid-log phase. The cells were fixed in $4 \%$ paraformaldehyde overnight, washed two times in $1 \times$ PBS and stored at $-80^{\circ} \mathrm{C}$ until use. For the whole-cell hybridization, we used the protocol described by Poulsen et al. ${ }^{13}$ with some modifications ${ }^{41}$. Briefly, fixed $E$. coli cells from the in vitro cultures were washed once with PBS, once with solution I (35\% formamide, $100 \mathrm{mM}$ Tris (pH 7.5), $0.1 \%$ SDS and $0.9 \mathrm{M} \mathrm{NaCl}$ ), and hybridized with a probe specific to E. coli $23 \mathrm{~S}$ rRNA (EC 1531; 5'-CACCGTAGTGCCTCGTCATCA-3') labelled with the fluorochrome $\mathrm{Cy} 3$ (final concentration $2.5 \mathrm{ng} \mu \mathrm{l}$ ). The hybridizations were carried for $16 \mathrm{~h}$ at $37^{\circ} \mathrm{C}$. The cells were then washed once with solution I and twice with solution II (35\% formamide, $100 \mathrm{mM}$ Tris ( $\mathrm{pH} 7.5)$ and $0.9 \mathrm{M} \mathrm{NaCl}$ ). Next, the cells were incubated for $15 \mathrm{~min}$ at $37^{\circ} \mathrm{C}$, centrifuged and resuspended in $1 \times$ PBS. Cy 3 fluorescence was measured by flow cytometry (LSR Fortessa, BD) and median fluorescence analysed with FlowJo v10. For each sample, two hybridizations were performed. The average of median fluorescence intensity of $\mathrm{Cy} 3$ was correlated with growth rate for each growth condition, thus enabling a measure of division time of $E$. coli.

WT and Rag $2^{-1-}$ mice $(n=6)$ were colonized with E. coli strain DM09 (ancestral) following the protocol described above for the evolution experiments. Faecal pellets were collected at days 1 and 3 after gavage, homogenized in $1 \times$ PBS and fixed in $4 \%$ paraformaldehyde overnight. Following fixation, cells were washed twice in $1 \times$ PBS and stored at $-80^{\circ} \mathrm{C}$ until use.

Whole-cell hybridization with the probe specific for E. coli $23 \mathrm{~S}$ rRNA was performed according to the protocol described before for the in vitro cultures. On the basis of the fluorescence intensity of the probe obtained with the hybridization and the previously obtained calibration curve, we inferred a given duplication time for E. coli colonizing WT and Rag2 ${ }^{-1-}$ mice (see Fig. 2b and Supplementary Fig. 4).

Comparison of $\boldsymbol{E}$. coli mutation rate between hosts. To estimate the equilibrium frequency for antibiotic resistance clones, we determined the fraction of $E$. coli clones carrying spontaneous resistance to different antibiotics. Slightly deleterious mutations conferring resistance to nalidix acid and rifampicin (point mutations in $g y r A$ and $r p o B$, respectively) or furazolidone (mutations in $n f_{s} A$, including IS insertions) are expected to spontaneously occur and be continuously eliminated by purifying selection in E. coli populations colonizing the mouse gut, hence resistance alleles are expected to reach a mutation-selection balance and their frequency to reach a stable equilibrium, which is proportional to the mutation rate.

Rag $^{-1-}$ and WT mice were colonized with E. coli strain DM08 (ancestral) following the protocol described above for the evolution experiments, and the frequency of spontaneous resistance was determined in faecal pellets collected over 15 days. We tested six animals of each genotype for rifampicin and nalidixic acid resistance and $10 \mathrm{Rag}^{-1-}$ and 11 WT mice for furazolidone resistance. Faecal pellets were collected, homogenized in $1 \times$ PBS and appropriate dilutions plated in LB agar supplemented with streptomycin $\left(100 \mu \mathrm{g} \mathrm{ml}^{-1}\right)$ or LB agar supplemented with streptomycin $\left(100 \mu \mathrm{g} \mathrm{ml}^{-1}\right)$ and nalidixic acid $\left(40 \mu \mathrm{g} \mathrm{ml}^{-1}\right)$, rifampicin $\left(100 \mu \mathrm{g} \mathrm{ml}^{-1}\right)$ or furazolidone $\left(1.25 \mu \mathrm{g} \mathrm{ml}^{-1}\right)$. The frequency of mutants resistant to each antibiotic was calculated as the ratio between the number of antibioticresistant clones and the total number of cells in each E. coli population. We also estimated the transposition frequency based on the fraction of furazolidone resistance mutants harbouring insertions in $n f_{s} A$.

In vivo competition fitness assays. We measured the relative fitness in vivo of an E. coli clone harbouring a IS insertion in the gat Z gene (clone $4 \mathrm{YFP}^{11}$ ) isolated from faecal samples after 24 days of adaptation to the gut of WT mice. This clone was competed either against the ancestral strain labelled with the opposite fluorescent marker (DM09) (Figs 2 and 3) or against a gatC mutant (derived from clone $12 \mathrm{YFP})^{11}$ carrying an insertion of $1 \mathrm{bp}$ in the gatC gene (Fig. 4).

The competitions were performed at a ratio of 1:1, over 3 days of cocolonization, following the same procedure described for the evolution experiments in SPF mice; with no streptomycin treatment for competitions carried out in GF mice. We estimated the selective advantage of $g a t Z$ over the ancestral from the slope of the linear regression of $\ln (\mathrm{gat} Z / \mathrm{anc})$ along time.
Microbiota analysis. To assess the gut microbiota composition of unmanipulated mice, we analysed faecal samples collected from five independently housed WT and $R a g 2^{-1-}$ mice belonging to different litters and in two experimental blocks $(3+2$ mice). These animals were bred and maintained in similar SPF conditions in the same barrier facility (see E. coli and mouse strains). With this experimental design, we aimed both to characterize a representative sample of the microbiota composition associated with each host genotype and minimize the cage effects that could bias our analysis.

To characterize the microbiotal context in which E. coli was evolving, we analysed faecal samples collected from WT and Rag ${ }^{-1-}$ mice at day 3 of the evolution experiment, that is, streptomycin-treated mice that had been colonized with $E$. coli for 3 days and housed individually. Similarly to unmanipulated animals, these mice were also bred and raised in SPF conditions but treated with streptomycin before and during colonization with E. coli (see Dynamics of adaptation of E. coli in the mouse gut above). We analysed a total of 10 mice of each genotype, with a minimum of 3 animals from each experimental block (WT: $\left.3+4+3 ; \operatorname{Rag}^{-1-}: 3+3+4\right)$.

The extraction was performed with a QIAamp DNA Stool Mini Kit (Qiagen), according to the manufacturer's instructions and an additional step of mechanical disruption ${ }^{21}$.

$16 \mathrm{~S}$ rRNA gene amplification and sequencing was carried out at the IGC Genomics Unit. Samples were amplified using primers specific to the V3-V4 region of the 16s rRNA gene and pair-end sequenced on an Illumina MiSeq Benchtop Sequencer, following Illumina recommendations. Samples were sequenced to a depth of at least 20,000 high-quality sequences. Reads were processed and analysed using mothur ${ }^{42}$ software, following the MiSeq $\mathrm{SOP}^{43}$ on the mothur wiki (http://www.mothur.org/wiki/MiSeq_SOP) with some modifications ${ }^{21}$. To minimize possible biases related with variable sequencing depth across samples, a subsampling of 20,000 high-quality reads was performed. Sequences were aligned in accordance with SILVA alignment ${ }^{44}$ and unique sequences were clustered into OTUs using a $97 \%$ identity cutoff. A phylogenetic tree was inferred using clearcut ${ }^{45}$ on the 16S rRNA sequence alignment generated by mothur. Alpha and beta measurements of diversity, both OTU- and phylogeny based, were obtained using mothur. The Chao ${ }^{46}$ and Shannon ${ }^{47}$ indices were used to estimate community richness and diversity, respectively. The phylogenetic diversity was calculated as the total of the unique branch length in the phylogenetic tree. As a measure of beta diversity we evaluated the structure of microbial communities, a parameter that takes into account not only the presence and absence of organisms but also their abundance. To assess similarities in community structure OTU- (Bray-Curtis ${ }^{48}$ ) or phylogeny-based (weighted UniFrac ${ }^{49}$ ) measures of beta diversity were calculated. Differences in phylogenetic community membership were evaluated using unweighted UniFrac ${ }^{50}$. PCoA on the distance matrices of the beta diversity metrics were performed using Mothur and R software package (http://www.R-project.org).

Haplotypic structure of $\mathbf{R a g 2}^{-1-}$ populations. To estimate the E. coli haplotypic frequencies evolving in the Rag $2^{-I-}$ mice, we analysed $\sim 20$ clones isolated from faecal pellets from the last day of the evolution experiment for each of the evolved lines. These clones were screened for the presence of mutations shown to be adaptive in WT animals ${ }^{11}$ : mutations in the gat operon, insertions of IS elements in the regulatory regions of $f o c A$ and $d c u B$ and point mutations in the coding region of $s r l R$. We typed gat mutants based on the inability to metabolize galactitol using the procedure described above for the dynamics of the gat-negative phenotype. The remaining mutations were identified by target PCR; insertions of IS elements were identified based on the increase in size of the PCR products and Sanger sequencing was used to detect SNPs. PCR reactions were performed in the same conditions and with primers as previously described ${ }^{11}$, but in a total volume of $12.5 \mu$ l.

WGS of $\boldsymbol{E}$. coli populations. To identify the pool of mutations segregating in $E$. coli populations evolved in Rag2 $2^{-1-}$ and WT mice, we performed WGS of a large sample of each population. We isolated more than 1,000 clones from mice faecal pellets from day 24 of evolution, and we extracted the DNA from this mixture of clones following a protocol previously described ${ }^{11}$. The DNA library construction and sequencing was carried out by the IGC genomics facility. Each sample was pair-end sequenced on an Illumina MiSeq Benchtop Sequencer. Standard procedures produced data sets of Illumina paired-end 250-bp read pairs. The mean coverage per sample was between $\times 100$ and $\times 180$ for both WT and Rag2 ${ }^{-1-}$. Subsampling of 3 million reads was performed to obtain an average of $\times 100$ coverage. Mutations were identified using the BRESEQ pipeline v0.23 with the polymorphism option on (see Supplementary Tables 6 and 7). The default settings were used except for: (a) requiring a minimum coverage of three reads on each strand per polymorphism; (b) elimination of polymorphism predictions occurring in homopolymers of length greater than 3 ; (c) elimination of polymorphism predictions with significant $(P$ value $<0.05)$ strand or base quality score bias. In addition, unassigned new junctions supported by a minimum of two reads in each direction and insertions of IS elements where only one new junction was identified were also accepted. A cutoff of $5 \%$ of frequency was used to identify mutations segregating at high frequency in the populations (Supplementary Tables 6 and 7). We defined parallel mutations (Table 1) as mutational events that occurred in a minimum of two animals (either WT or $\mathrm{Rag}^{-l-}$ ) and that reached a minimum frequency of $10 \%$ in at least one population. 
Statistical analyses. All statistical analyses were performed in R software: http:// www.r-project.org/.

To test for differences in the initial deviation of the fluorescent marker, we used a binomial test that compared the number of WT and Rag $2^{-l-}$ lines in which the slope of the linear regression of $\ln (\mathrm{YFP} / \mathrm{CFP})$ deviated significantly from 0 on the first 6 days of colonization.

We used a linear mixed model on the logarithm of bacterial counts per gram of faeces, with host genotype and time as fixed effects and individuals as random effects to compare the temporal dynamics of bacterial loads between Rag $^{-1-}$ and WT mice. To test whether the bacterial loads over time were affected by host genotype, we contrasted a model with the interaction between these two effects with a model lacking this interaction. Additionally, we also tested if the bacterial loads differed globally between the two genotypes, by comparing models including or excluding the host genotype as a fixed effect.

To assess the influence of host genotype on the temporal dynamics of the gatnegative phenotype, we employed a generalized linear mixed model, with host genotype and time as fixed effects and individuals as random effects. The gat frequencies were weighted by the number of counted colony-forming units, and the initial frequency was constrained to the same value in WT and Rag2 ${ }^{-1-}$ dynamics.

Mann-Whitney $U$-tests were used to evaluate differences on the estimated generation time or mutation frequency between WT and Rag2 $2^{-1-}$ mice.

ANOVA was used to investigate the impact of the microbiota and the immune state on the selective advantage of the gatZ mutation, followed by Tukey's post hoc tests to assess differences between groups. Two-tailed F-tests were used to determine whether the variance for the selective advantage of $g a t Z$ was increased in Rag2 ${ }^{-1-}$ compared with WT animals.

Mann-Whitney $U$-tests were used to identify genera differentially represented in WT or Rag $2^{-l}$ mice. The $P$ values were adjusted for multiple comparisons using the Benjamini and Hochberg correction. Two-tailed F-tests were used to determine differences in the variance of the genera.

To determine whether the parallel mutations identified by WGS were more represented in one host that in the other, we performed binomial tests.

Ethics statement. All experiments involving animals were approved by the Institutional Ethics Committee at the Instituto Gulbenkian de Ciência (project no. A009/2010 with approval date 15 October 2010), following the Portuguese legislation (PORT 1005/92), which complies with the European Directive 86/609/EEC of the European Council.

\section{References}

1. Ivanov, I. I. et al. Induction of intestinal Th17 cells by segmented filamentous bacteria. Cell 139, 485-498 (2009).

2. Atarashi, K. et al. Induction of colonic regulatory $\mathrm{T}$ cells by indigenous Clostridium species. Science 331, 337-341 (2011).

3. Round, J. L. \& Mazmanian, S. K. Inducible Foxp3 + regulatory T-cell development by a commensal bacterium of the intestinal microbiota. Proc. Natl Acad. Sci. USA 107, 12204-12209 (2010).

4. Ridaura, V. K. et al. Gut microbiota from twins discordant for obesity modulate metabolism in mice. Science 341, 1241214 (2013).

5. Cammarota, G., Ianiro, G. \& Gasbarrini, A. Fecal microbiota transplantation for the treatment of Clostridium difficile infection: a systematic review. J. Clin. Gastroenterol. 48, 693-702 (2014).

6. Kawamoto, S. et al. Foxp3(+) T cells regulate immunoglobulin A selection and facilitate diversification of bacterial species responsible for immune homeostasis. Immunity 41, 152-165 (2014).

7. Zhang, H., Sparks, J. B., Karyala, S. V., Settlage, R. \& Luo, X. M. Host adaptive immunity alters gut microbiota. ISME J. 9, 770-781 (2015).

8. Ley, R. E., Lozupone, C. A., Hamady, M., Knight, R. \& Gordon, J. I. Worlds within worlds: evolution of the vertebrate gut microbiota. Nat. Rev. Microbiol. 6, 776-788 (2008).

9. Mukherjee, P. K. et al. Mycobiota in gastrointestinal diseases. Nat. Rev. Gastroenterol. Hepatol. 12, 77-87 (2015).

10. Sartor, R. B. \& Mazmanian, S. K. Intestinal microbes in inflammatory bowel diseases. Am. J. Gastroenterol. Suppl. 1, 15-21 (2012).

11. Barroso-Batista, J. et al. The first steps of adaptation of Escherichia coli to the gut are dominated by soft sweeps. PLoS Genet. 10, e1004182 (2014).

12. Charlesworth, B. Elements of Evolutionary Genetics (Roberts and Company Publishers, 2010).

13. Poulsen, L. K., Licht, T. R., Rang, C., Krogfelt, K. A. \& Molin, S. Physiological state of Escherichia coli BJ4 growing in the large intestines of streptomycintreated mice. J. Bacteriol. 177, 5840-5845 (1995).

14. Whiteway, J. et al. Oxygen-insensitive nitroreductases: analysis of the roles of $\mathrm{nfsA}$ and $\mathrm{nfsB}$ in development of resistance to 5-nitrofuran derivatives in Escherichia coli. J. Bacteriol. 180, 5529-5539 (1998).

15. Elinav, E., Thaiss, C. A. \& Flavell, R. A. Analysis of microbiota alterations in inflammasome-deficient mice. Methods Mol. Biol. 1040, 185-194 (2013).
16. Dimitriu, P. A. et al. Temporal stability of the mouse gut microbiota in relation to innate and adaptive immunity. Environ. Microbiol. Rep. 5, 200-210 (2013).

17. Scholz, F., Badgley, B. D., Sadowsky, M. J. \& Kaplan, D. H. Immune mediated shaping of microflora community composition depends on barrier site. PLoS ONE 9, e84019 (2014).

18. Suzuki, K. et al. Aberrant expansion of segmented filamentous bacteria in IgA-deficient gut. Proc. Natl Acad. Sci. USA 101, 1981-1986 (2004).

19. Thoene-Reineke, C. et al. Composition of intestinal microbiota in immunedeficient mice kept in three different housing conditions. PLoS ONE 9, el13406 (2014).

20. Shulzhenko, N. et al. Crosstalk between B lymphocytes, microbiota and the intestinal epithelium governs immunity versus metabolism in the gut. Nat. Med. 17, 1585-1593 (2011)

21. Thompson, J. A., Oliveira, R. A., Djukovic, A., Ubeda, C. \& Xavier, K. B. Manipulation of the quorum sensing signal AI-2 affects the antibiotic-treated gut microbiota. Cell Rep. 10, 1861-1871 (2015).

22. Leatham, M. P. et al. Mouse intestine selects nonmotile flhDC mutants of Escherichia coli MG1655 with increased colonizing ability and better utilization of carbon sources. Infect. Immun. 73, 8039-8049 (2005).

23. Leatham-Jensen, M. P. et al. The streptomycin-treated mouse intestine selects Escherichia coli envZ missense mutants that interact with dense and diverse intestinal microbiota. Infect. Immun. 80, 1716-1727 (2012).

24. Murray, E. L. \& Conway, T. Multiple regulators control expression of the Entner-Doudoroff Aldolase (Eda) of Escherichia coli. J. Bacteriol. 187, 991-1000 (2005).

25. Peekhaus, N. \& Conway, T. What's for dinner?: Entner-Doudoroff metabolism in Escherichia coli. J. Bacteriol. 180, 3495-3502 (1998).

26. Stratmann, T., Madhusudan, S. \& Schnetz, K. Regulation of the yjjQ-bglJ operon, encoding LuxR-Type transcription factors, and the divergent yjjP gene by H-NS and LeuO. J. Bacteriol. 190, 926-935 (2008).

27. Lin, H.-Y., Bledsoe, P. J. \& Stewart, V. Activation of yeaR-yoaG operon transcription by the nitrate-responsive regulator NarL is independent of oxygen- responsive regulator Fnr in Escherichia coli K-12. J. Bacteriol. 189, 7539-7548 (2007).

28. Georgellis, D., Kwon, O. \& Lin, E. C. C. Quinones as the redox signal for the arc two-component system of bacteria. Science 292, 2314-2316 (2001).

29. Wiame, E., Delpierre, G., Collard, F. \& Schaftingen, E. V. Identification of a pathway for the utilization of the amadori product fructoselysine in Escherichia coli. J. Biol. Chem. 277, 42523-42529 (2002).

30. Yoshikawa, A., Isono, S., Sheback, A. \& Isono, K. Cloning and nucleotide sequencing of the genes rimI and rimJ which encode enzymes acetylating ribosomal proteins S18 and S5 of Escherichia coli K12. Mol. Gen. Genet. 209, 481-488 (1987).

31. Ubeda, C. et al. Intestinal microbiota containing Barnesiella species cures vancomycin-resistant Enterococcus faecium colonization. Infect. Immun. 81, 965-973 (2013).

32. Fransen, F. et al. BALB/c and C57BL/6 mice differ in polyreactive IgA abundance, which impacts the generation of antigen-specific IgA and microbiota diversity. Immunity 43, 527-540 (2015).

33. Faith, J. J., Ahern, P. P., Ridaura, V. K., Cheng, J. \& Gordon, J. I. Identifying gut microbe-host phenotype relationships using combinatorial communities in gnotobiotic mice. Sci. Transl. Med. 6, 220ra11 (2014).

34. Engel, P., Krämer, R. \& Unden, G. Transport of C4-dicarboxylates by anaerobically grown Escherichia coli. Energetics and mechanism of exchange, uptake and efflux. Eur. J. Biochem. 222, 605-614 (1994).

35. Ferreyra, J. A. et al. Gut microbiota-produced succinate promotes C. difficile infection after antibiotic treatment or motility disturbance. Cell Host Microbe 16, 770-777 (2014).

36. Jones, S. A. et al. Anaerobic respiration of Escherichia coli in the mouse intestine. Infect. Immun. 79, 4218-4226 (2011).

37. Chang, D. E. et al. Carbon nutrition of Escherichia coli in the mouse intestine. Proc. Natl Acad. Sci. USA 101, 7427-7432 (2004).

38. Sweeney, N. J., Laux, D. C. \& Cohen, P. S. Escherichia coli F-18 and E. coli K-12 eda mutants do not colonize the streptomycin-treated mouse large intestine. Infect. Immun. 64, 3504-3511 (1996).

39. Blattner, F. R. et al. The complete genome sequence of Escherichia coli K-12. Science 277, 1453-1462 (1997).

40. Conway, T., Krogfelt, K. A. \& Cohen, P. S. in EcoSal-Escherichia coli and Salmonella: cellular and molecular biology (ed. Curtiss, R.) Ch. 8.3.1.2 (ASM Press, 2004).

41. Skinner, S. O., Sepúlveda, L. A., Xu, H. \& Golding, I. Measuring mRNA copy number in individual Escherichia coli cells using single-molecule fluorescent in situ hybridization. Nat. Protoc. 8, 1100-1113 (2013).

42. Schloss, P. D. et al. Introducing mothur: open-source, platform-independent, community-supported software for describing and comparing microbial communities. Appl. Environ. Microbiol. 75, 7537-7541 (2009). 
43. Schloss, P. D., Gevers, D. \& Westcott, S. L. Reducing the effects of PCR amplification and sequencing artifacts on 16S rRNA-based studies. PLoS ONE 6, e27310 (2011).

44. Pruesse, E. et al. SILVA: a comprehensive online resource for quality checked and aligned ribosomal RNA sequence data compatible with ARB. Nucleic Acids Res. 35, 7188-7196 (2007).

45. Sheneman, L., Evans, J. \& Foster, J. A. Clearcut: a fast implementation of relaxed neighbor joining. Bioinforma. Oxf. Engl 22, 2823-2824 (2006).

46. Chao, A. Nonparametric estimation of the number of classes in a population. Scand. J. Stat. 11, 265-270 (1984).

47. Shannon, C. E. A mathematical theory of communication. Bell Syst. Tech. J. 27, 379-423 (1948).

48. Bray, J. R. \& Curtis, J. T. An ordination of the upland forest communities of southern Wisconsin. Ecol. Monogr. 27, 326-349 (1957).

49. Lozupone, C. A., Hamady, M., Kelley, S. T. \& Knight, R. Quantitative and qualitative $\beta$ diversity measures lead to different insights into factors that structure microbial communities. Appl. Environ. Microbiol. 73, 1576-1585 (2007).

50. Lozupone, C., Lladser, M. E., Knights, D., Stombaugh, J. \& Knight, R. UniFrac: an effective distance metric for microbial community comparison. ISME J. 5, 169-172 (2011).

\section{Acknowledgements}

We thank A. Sousa and J. Sousa for help in the sequence analysis and continuous discussions along the work, R. Ramiro and N. Martins for help in the statistical analysis, R. Oliveira and R. Areal for help with the microbiota analysis and V. Barreto, J.

Thompson and Gordo's lab members for critically reading the manuscript. The research leading to these results has received funding from the European Research Council under the European Community's Seventh Framework Programme (FP7/2007-2013)/ERC grant agreement no. 260421-ECOADAPT and from the Portuguese Science Foundation FCT (PTDC/BIA-EVF/118075/2010). We thank the IGC animals house Unit and the gnotobiology service partially funded through FCT grant RECI/IMI-IMU/0038/2012 and EU-FP7 infrastructure grants EMMA and InfrafrontierI3. I.G. acknowledges salary support of LAO/ITQB \& FCT. J.B.-B. was supported by grant SFRH/BD/80257/2011 from FCT.

\section{Author contributions}

I.G., J.D. and J.B.-B. conceived and designed the experiments. J.B.-B. performed the experiments. J.B.-B., I.G. and J.D. analysed the data. I.G. and J.D. contributed reagents/ materials/analysis tools. J.B.-B., I.G. and J.D. wrote the paper. The funders had no role in the study design, data collection and analysis, decision to publish or preparation of the manuscript.

\section{Additional information}

Accession codes: Genome sequencing and 16S rRNA gene sequencing data have been deposited in the NCBI Read Archive database with accession code PRJNA297801.

Supplementary Information accompanies this paper at http://www.nature.com/ naturecommunications

Competing financial interests: The authors declare no competing financial interests.

Reprints and permission information is available online at http://npg.nature.com/ reprintsandpermissions/

How to cite this article: Barroso-Batista, J. et al. Adaptive immunity increases the pace and predictability of evolutionary change in commensal gut bacteria. Nat. Commun. 6:8945 doi: 10.1038/ncomms9945 (2015).

\section{(c) (1)}

This work is licensed under a Creative Commons Attribution 4.0 International License. The images or other third party material in this article are included in the article's Creative Commons license, unless indicated otherwise in the credit line; if the material is not included under the Creative Commons license, users will need to obtain permission from the license holder to reproduce the material. To view a copy of this license, visit http://creativecommons.org/licenses/by/4.0/ 